\title{
A Reading History Logger for Supporting Reading Habit Development
}

\author{
Yasuo Miyoshi ${ }^{1}$ and Takaaki Oobayashi ${ }^{2}$ \\ ${ }^{1}$ Faculty of Science, Kochi University, Japan \\ ${ }^{2}$ Graduate School of Integrated Arts and Sciences, Kochi University, Japan \\ \{miyoshi, obayashi\}@is.kochi-u.ac.jp
}

\begin{abstract}
We are developing a social networking site for the purpose of support for a reading habit development. To support the habit development, it is necessary to grasp the state of the user's reading in real time. However, letting a user register every reading history is a big burden for the user aiming at the habit development. Therefore we have developed a bookmark-style reading history logger device using Eye-Fi and PSoC. The bookmark device has a function to register the reading date/time information to any photo-sharing site. Our social networking site for a habit development can get the reading time by checking a web feed of the photo-sharing site.
\end{abstract}

Keywords: Habit Development, Reading, Logger, Peer Mentoring.

\section{Introduction}

Development of learning habit and improvement of learning motivation are important in self-directed learning activity. Activity of reading books is a one of self-directed learning activity. We focus peer mentoring[1] utilizing interaction-centered model in motivational design[2], and intend to design an environment for activities of peer mentoring. We aim to support development of learning habit with peer mentoring, and are developing an environment for peer mentoring on a social networking site. Gathering a state of the users is required to peer mentoring. However, it is nonsense that the user who wants to develop the reading habit records the history at every reading. Therefore our study considers a method to get the reading histories in some electronic book readers (e.g. Kindle, iPad) and traditional paper media books [3][4].

\section{Reading Habit Development with Peer Mentoring}

We have focused on a peer mentoring method which habit development applicants make mentoring with each other. Figure 1 shows peer mentoring. The applicant must observe a partner and grasp the state of the partner to comment to the partner in order to perform mentoring. However, it is impossible that the applicant always observe the state of reading of the partner in the case that the object of habit development is reading. They cannot give them appropriate advices with each other. Therefore we 
have proposed a peer mentoring system on a social networking site as shown in figure 2. The habit development applicant records one's reading history in the system as log instead of observing the reading state of the partner and record. The system analyzes the $\log$ and measures the appropriate timing for advising. If the appropriate timing comes, the system shows the reading state of the partner and gets the applicant advises the partner. We know that when learners are conscious of the existence of their familiar person, they can maintain their motivation in early learning activity[5]. The system does not show some automatic generated sentences as advice to the applicant because the repetition of generated advices without warmth negatively influence their motivations.

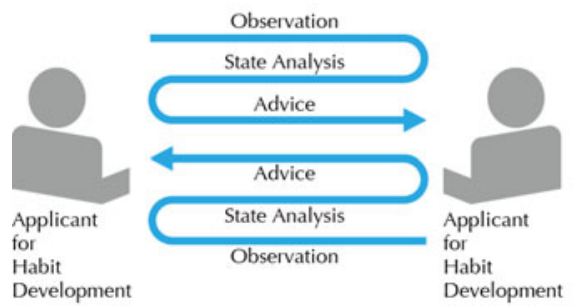

Fig. 1. Peer Mentoring

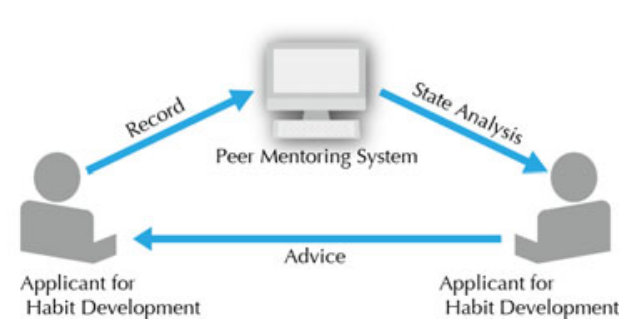

Fig. 2. Peer Mentoring System

\section{Reading History}

\subsection{Reading History for Supporting Reading Habit Development}

Ideally, we should get the reading history which has Five W's and One H. Example: \{Who: Ichiro Tanaka. When: one hour from 19:00 on $7^{\text {th }}$ July 2010. What:

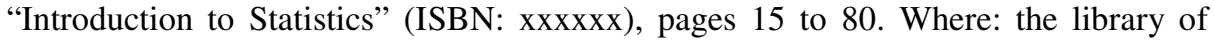
xxxx University. Why: to write a report for the lecture of statistics. How: while taking the memo.\} For supporting reading habit development, the required information should be for confirming whether or not the user read a book. That is we must get the user information (who) and time information (when). The requirements for getting time information are able to get start (restart) and finish (abort) time automatically and promptly.

\subsection{To Get a Reading History}

We consider to get reading history in paper media books, because electronic book readers are unfamiliar and there are few digitalize book data. It is difficult to get a reading history except time information from paper media. We propose a bookmark-style reading history logging device for getting automatically the start time and the finish time. This device is used like an original bookmark and held between the pages of a book. It detects opening and shutting of the book and records the time as start time and finish time of reading. Therefore it can record automatically the time of reading without user's operation. Furthermore it can get the information promptly if it uses a storage that has a wireless communication function. 


\section{Prototyping a Bookmark-Style Reading History Logging Device}

We have prototyped a bookmark-style reading history logging device for getting a reading history on a paper media book. It detects opening and shutting of the book by an optical sensor (CdS cell). The end of the sensor connects with tied optical fiber because the sensor has thickness. As shown in figure 3, a bundle of optical fiber is laid slimly and closely together. This thin part is tucked into the book. This bookmark device does not detect a light while into the shut book, but can detect it while opening the book. At this time, the microcontroller writes the time, as the reading time, onto Eye-Fi SD Card. The microcontroller embeds the reading time, as Exif metadata format, in a dummy picture file, because the Eye-Fi has a function to upload

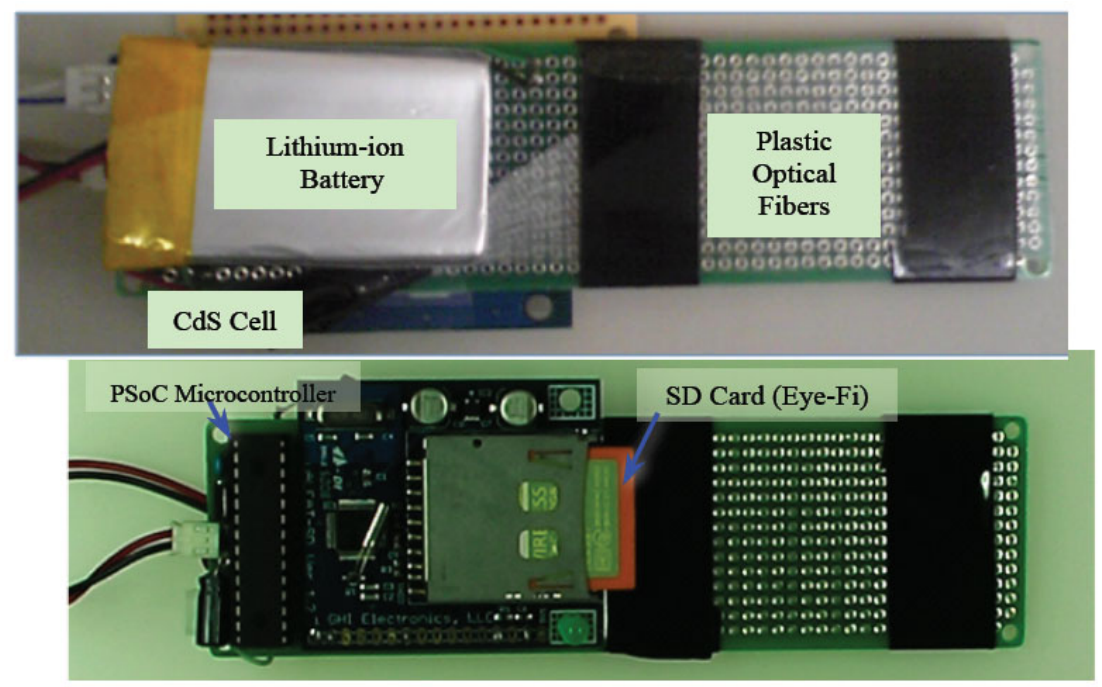

Fig. 3. Reading History Logging Device (front side and back side)

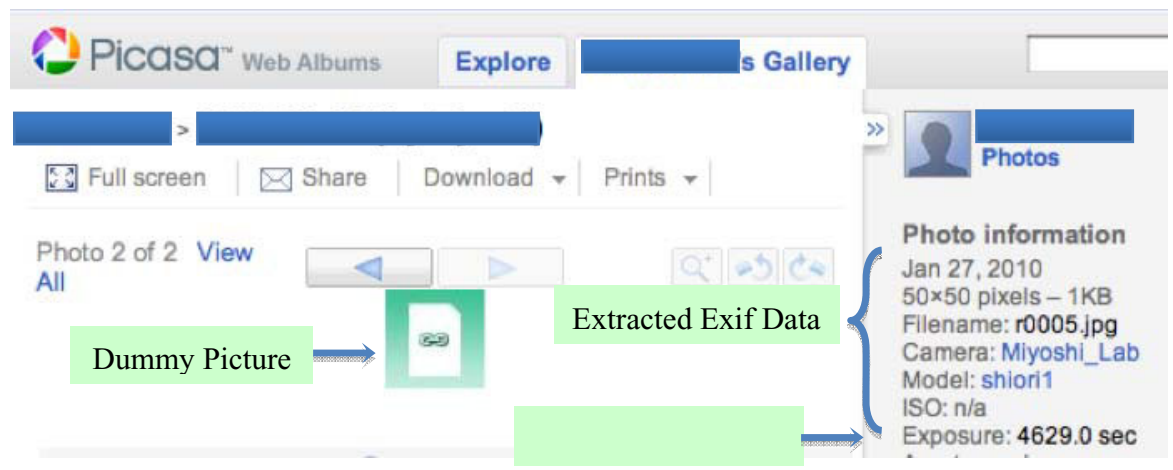

Fig. 4. Extracted Exif Data from a Dummy Picture on a Photo-Sharing Site 
wirelessly and automatically photo files. When the dummy picture including the reading time is written in the Eye-Fi, in the enabled wireless LAN area, the Eye-Fi card uploads the picture to any supported photo-sharing website automatically. The photo-sharing site that received the dummy picture should probably be able to extract the Exif data from the dummy picture (figure 4). It can be got the reading time information that is included in Exif data by checking a web feed of the photo-sharing site.

\section{Summary}

Although our prototyping device can only record user's reading time, it is used like an original bookmark, and can record automatically the time of reading without user's operation. We have confirmed that our social networking site that is for supporting reading habit development can get automatically users' reading histories that were recorded by the prototyping device. We think that data logging using Eye-Fi like our method is useful for other data logging like a lifelog.

\section{References}

1. Baker, W., McNicoll, A.: I can get by with a little help from my friends: Peer mentoring critical friends for the reflective practitioner. In: Proc. of the 2006 Ann. Intr. Conf. of HERDSA, pp. 28-34 (2006)

2. Keller, J.M.: Motivational design. In: Unuwin, C. (ed.) Encyclopaedia of Educational Media Communications and Technology, 2nd edn., pp. 406-409. Greenwood Press, Westport (1988)

3. Amazon.com, Inc.: Kindle Development Kit for Active Content, https://kindlepublishingamazon. com/gp/vendor/members/kindlepu bs/kdk/home

4. Apple Inc.: Start Developing iPad Apps, http: / / developer.apple.com/ipad/sdk/

5. Miyoshi, Y., Okamoto, R., Kanenishi, K., Yano, Y.: A Design of Social Networking Service for Supporting Learning Habits Development. In: Proc. of IADIS International Conference Cognition and Exploratory Learning in Digital Age (CELDA 2009), pp. 396399 (2009) 